\title{
Massive Star Evolution: Binaries as Two Single Stars
}

\author{
Cyril Georgy and Sylvia Ekström ${ }^{a}$
}

\begin{abstract}
Binary stars are of course more than two stars, but they are also at least two stars. In this chapter we will review some aspects of the physics governing the evolution of single massive stars. We will also review the uncertainties of key physical ingredients: mass loss, rotation and convection.
\end{abstract}

\subsection{The basics}

Massive stars are stars more massive than about $8-10 M_{\odot}$, that are bound to die in the explosion of a supernova or collapsing directly into a black hole (Heger et al., 2003, Jones et al. 2013). Their life is marked by the succession of all burning stages up to silicon burning.

The modelling of massive stars is done by the mean of one-dimension (1d) stellar evolution codes (see below), which solve the four equations of stellar structure (see Kippenhahn and Weigert, 1990, for a classical textbook), coupled with a network of nuclear reactions providing the energy generation rate inside the stellar model, and the time evolution of the chemical content of the star. On top of that, various effects can be accounted for, such as rotation or mass loss, particularly important in the context of the evolution of massive stars.

An important aspect of massive star evolution that became prominent during the past decade is that a significant fraction of massive stars are found in multiple systems. Large surveys in different environments suggest that $50 \%$ to $70 \%$ of all massive stars (Sana et al. 2012. 2013) are in binary (or multiple) systems where components are sufficiently close to interact at least once during their lifetime. Evolution of massive stars in multiple systems can be very different from single star evolution (van den Heuvel, 1975;, Vanbeveren, 1991; Podsiadlowski et al., 1992, Vanbeveren et al., 1998; Eldridge et al., 2008; de Mink et al.

\footnotetext{
${ }^{a}$ The authors acknowledge support from the Swiss National Science Foundation (project number 200020-172505). CG acknowledge PRACE for awarding access to resource MareNostrum 4 based in Spain at Barcelona Supercomputing Center. The support of David Vicente and Janko Strassburg from Barcelona Supercomputing Center, Spain, to the technical work is gratefully acknowledged.
} 
2013, among others). Taking multiplicity into account is thus extremely important when dealing with massive star populations.

It is however important to keep in mind that it is utopian to model correctly multiple stellar systems without being able to accurately describe the evolution of a single star. In this chapter, we discuss uncertainties of key physical processes taking place in single star models and their consequences. These shortcomings should be kept in mind by anybody using results from stellar evolution computations or from population synthesis.

\subsection{Mass loss}

Mass loss is a very important ingredient to be included in stellar evolution code when dealing with massive stars. At solar metallicity, a $15 M_{\odot}$ star loses 2 to $3 M_{\odot}$ during its entire life (but only a few tenths of a solar mass during its main sequence). At the other extremity of the mass spectrum, a $120 M_{\odot}$ star loses up to $100 M_{\odot}$ during its lifetime, including several tens during the main sequence (see e.g. Ekström et al. 2012). The situation is even more extreme in the case of very massive stars, which lose most of their mass in the course of their life (Yusof et al., 2013).

During the main sequence phase, winds from massive stars are in general described in the framework of the radiation-driven wind theory ("CAK" theory, see Lucy and Solomon. 1970: Castor et al. 1975). It has been improved over the years to accommodate for supplementary processes such as multiple scattering, wind clumping, etc. (see the review by Puls et al. 2008). It has lead to the computation of theoretical mass-loss rates that are extensively used in stellar evolution calculations (Vink et al., 2000, 2001). However, the face-values of the obtained mass-loss rates computed with this recipe seems to disagree by a factor 2-3 with mass-loss rates obtained observationally at different wavelengths (e.g. Najarro et al., 2011; Šurlan et al. 2013, Rauw et al. 2015). This uncertainty on the massloss rates that should be adopted in stellar evolution models has a major impact on the results of such simulations. As an illustration, Fig. 1.1 shows tracks in the HertzsprungRussell diagram for two $60 M_{\odot}$ models with different prescriptions for the computation of the mass-loss rates on the main-sequence: a model using Vink et al. (2000, solid line) and one using Kudritzki and Puls (2000, dotted line). Mass-loss rates during the advanced stages are kept the same (see also Keszthelyi et al., 2017). It shows that a difference of a factor of two in the mass-loss rates during the main sequence modifies completely the late evolution and the end-point just before the supernova.

For the most massive $\mathrm{O}$ and Wolf-Rayet (WR) stars, a dependence of the mass loss rates on the Eddington factor $\Gamma_{\mathrm{Edd}}=\frac{\kappa L}{4 \pi c G M}$ seems to appear when they reach large $\Gamma_{\text {Edd }}$ Vink, 2006; Vink et al. 2011; Gräfener et al., 2011; Bestenlehner et al., 2014). This dependence is around $\Gamma_{\mathrm{Edd}}{ }^{2}$ for O-stars with $\Gamma_{\mathrm{Edd}}<0.70$, and around $\Gamma_{\mathrm{Edd}}{ }^{4}$ for WR stars with $\Gamma_{\mathrm{Edd}}>$ 0.70 .

Mass loss rates usually scale with the metallicity, but the scaling to adopt is not yet completely clear. The $Z$ dependence has been estimated initially to be 0.4 by Abbott (1982), 


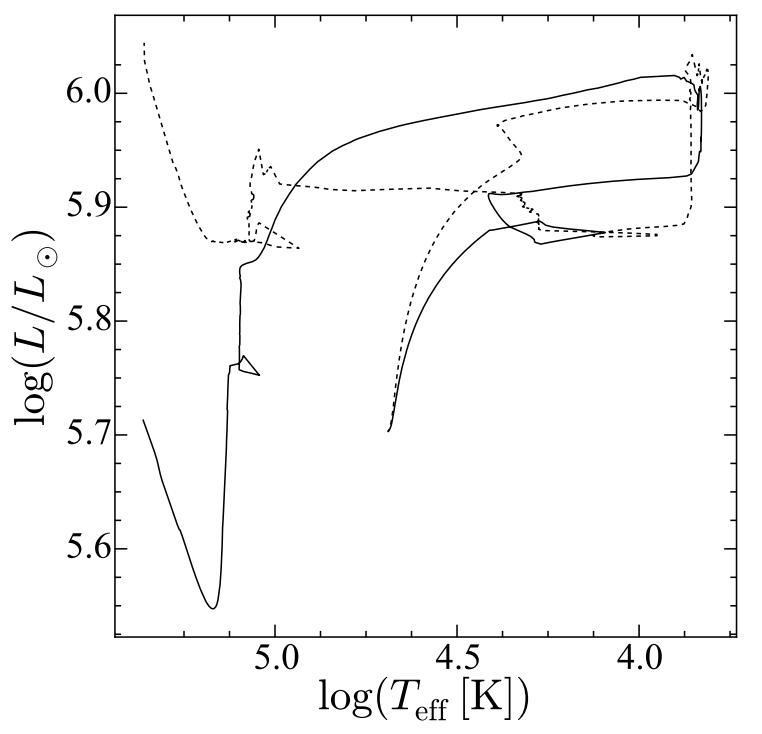

Figure 1.1 Tracks in the Hertzsprung-Russell diagram of a $60 M_{\odot}$ model computed with two different prescriptions for the mass-loss rates during the main-sequence: Vink et al. (2000, solid line) and Kudritzki and Puls (2000, dotted line). The impact on the tracks is clearly visible, due to the much higher rates with the Vink et al. (2000) prescription.

and then between 0.5 (Kudritzki, 2002) and 0.85 (Vink et al., 2001). Mokiem et al. (2007) find the empirical scaling deduced from observations of early B-type and O-type stars to be 0.83 for a smooth wind solution, or 0.72 when a correction for clumping is applied.

During the red supergiant phase, the mass-loss rates are even less well known. Observational determinations exhibit a very strong scatter, with variations over more than 2 orders of magnitude at a given luminosity (Mauron and Josselin, 2011; van Loon et al., 2005; Beasor and Davies, 2016, 2017, Georgy, 2017). As the winds from red supergiants are still not well understood from a theoretical point of view, the current prescriptions used in stellar evolution code are fits to these observational data. Questions such as: "in what kind of star, when and how long do the heavy mass-loss episodes occur? What is the average mass-loss rate at a given evolutionary stage?" need to be answered in order to correctly model the late stages of stellar evolution.

In case the mass-loss rates are best described by the prescriptions leading to rates in the low range of the observations, stars up to about $30 M_{\odot}$ end their life as a red supergiant. In such case, explanations to the so-called "red supergiant problem" (Smartt et al. 2009) have to be found. Strong extinction of the supernova event due to the dust in the strong winds of the more massive red supergiants (Walmswell and Eldridge, 2012) or direct collapse into a black hole (Adams et al., 2017) have been suggested. In case average mass-loss rates during the red supergiant phases are underestimated in usual prescriptions, it allows the star 
to evolve back toward the hot side of the Hertzsprung-Russell diagram (Vanbeveren et al. 1998, Georgy, 2012, Meynet et al., 2015). In this context, it is also interesting to note that a theoretical explanation of the pulsating properties of a class of variable blue supergiants, the " $\alpha$-Cygni" variables, seems to require that these stars have lost their hydrogen-rich envelope through a strong mass-loss episode, possibly during a previous red supergiant stage (Saio et al. 2013).

\subsection{Internal mixing processes}

Mixing processes are any physical process able to transport chemical elements or angular momentum from one place to another inside a star. They are important as they can considerably affect the way a star evolves, as well as its surface (and observable) properties. Historically, the first of such process included in the modelling of stars was convection (even before people started considering heat transfer by radiation, see Eddington, 1926). Rotation is another such process and has been studied very early during the 20th century von Zeipel, 1924, Eddington, 1925: Endal and Sofia, 1976). In this chapter, we focus only on these two processes (each of them would still require a whole book for an in depth description, like Maeder 2009, for rotation), and we will not consider other mixing processes such as radiative diffusion, gravitational settling, internal waves, magnetic field, or tides, among others.

\subsubsection{Convection}

The treatment of convection in classical $1 \mathrm{~d}$ stellar evolution codes is a long standing problem of stellar evolution (e.g. Arnett et al., 2015). In most of stellar evolution codes (e.g. the Geneva Stellar Evolution code, Eggenberger et al. 2008, MESA, Paxton et al. 2011. 2013, 2015, 2017, STERN, Petrovic et al. 2005, KEPLER, Heger et al.|2000; FRANEC, Chieffi and Limongi|2013 for the most widely used code in the massive star community), it is a two steps process: 1 ) determine the boundaries of the convective zones, often using the "Schwarzschild" or "Ledoux" criterion (e.g. Kippenhahn and Weigert, 1990), and possibly extend them empirically to account for the "overshoot" (Maeder, 1975) which cannot be predicted by the linear perturbation theory used to elaborate the stability criteria; 2) compute the thermal gradient inside the convective zone, often using the "mixing-length theory" (MLT) or one of its derivative (Böhm-Vitense, 1958). This was devised before the publication in the west of Kolmogorov's work on turbulent cascade (Kolmogorov, 1941. 1962), and may be inconsistent with that now well-established theory (see also Arnett et al. 2009).

There are a variety of ways of implementing convection in 1d stellar evolution codes, following theoretical works or guided by hydrodynamics simulations (Zahn, 1991, Freytag et al., 1996; Meakin and Arnett, 2007). Recent works have shown how sensitive the out- 
puts of $1 \mathrm{~d}$ stellar modelling are to the detailed implementation of convection (Martins and Palacios, 2013, Georgy et al., 2014b).

In this context, hydrodynamics simulations of convection in stellar interiors are very important, as they allow one to study the behaviour of the flow inside the convective regions from first principles. With the rapid increase of computational resources during the past years and the development of efficient parallelised hydrodynamics code, it has become possible to perform such simulations in different regimes: convective envelopes of cool stars (e.g. Freytag and Höfner, 2008, Chiavassa et al., 2009, Viallet et al., 2013, Magic et al., 2013), intermediate helium-burning shell in intermediate mass stars (Herwig et al., 2006; Woodward et al. 2015), or late burning stages of massive star life, such as carbon-burning shell (Cristini et al., 2017), oxygen-burning shell (Meakin and Arnett. 2007, Müller et al., 2016, Jones et al., 2017), or silicon-burning shell (e.g. Couch et al., 2015). These simulations provide very important informations about the physics of the convective boundary (shape, location, motion, boundary mixing) and the interaction between the nuclear burning and the convection (see Fig. 1.2, where the chemical abundances of ${ }^{16} \mathrm{O},{ }^{20} \mathrm{Ne},{ }^{24} \mathrm{Mg}$, and ${ }^{28} \mathrm{Si}$ with respect to the centre of the convective zone are shown in the case of the simulation of a neon-burning shell). The results of such simulations can be used to deduce prescriptions for the convective boundary mixing (Freytag et al. 1996, Herwig, 2000, Jones et al. 2017). Using specific averaging techniques ("RANS" approach, see e.g. Viallet et al., 2013, Mocák et al. 2014), they are also useful in providing a new theoretical framework and new algorithms for the treatment of convection in 1d stellar evolution code (Arnett) et al. 2015.

The need for a new treatment of convection and convective boundary mixing is observationally motivated by the asteroseismic data available for main-sequence B-type stars (Moravveji et al. 2015), or from nucleosynthesis considerations at different production sites (Battino et al. 2016; Choplin et al. 2017). The modelling of convection has also a deep impact on the final structure of the star, and can considerably affect its final fate by changing its compactness (supernova explosion or not, and the type of remnant left behind, see O'Connor and Ott, 2011; Sukhbold and Woosley, 2014, Davis et al., 2017).

\subsubsection{Rotation}

Rotation has two different ways of influencing stellar evolution. 1) It creates a deformation of the star's shape such that the stellar characteristics become dependent on the colatitude considered, affecting the stellar parameters deduced from observations. It also induces an anisotropy in the mass lost by the star. 2) It triggers an internal mixing that transports chemicals and angular momentum, and induces many deviations from the standard nonrotating evolution. We detail both effects below. 


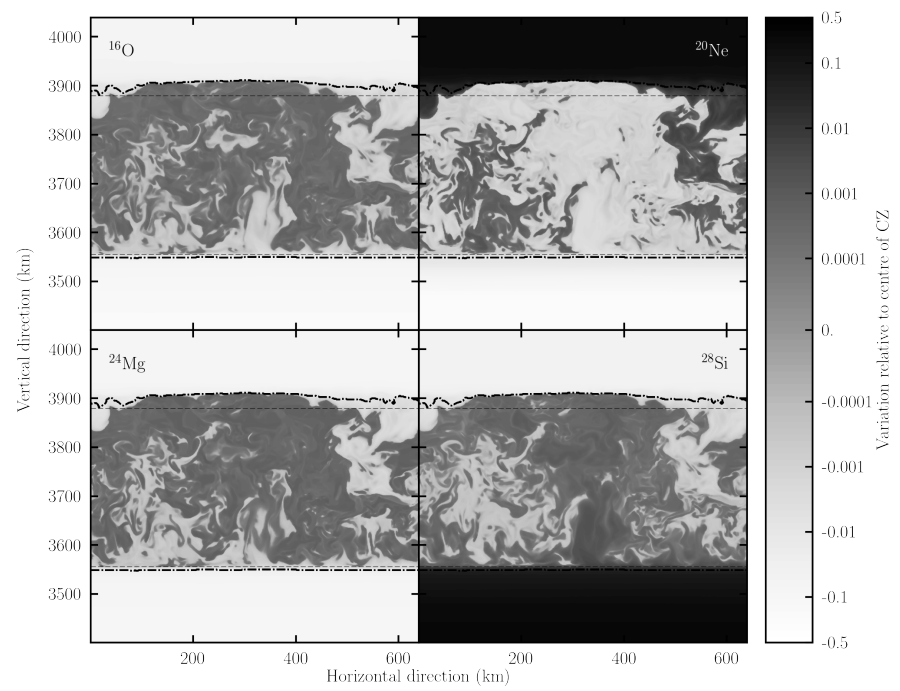

Figure 1.2 Abundance of ${ }^{16} \mathrm{O}$ (top-left panel), ${ }^{20} \mathrm{Ne}$ (top-right panel), ${ }^{24} \mathrm{Mg}$ (bottom-left panel), and ${ }^{28} \mathrm{Si}$ (bottom-right panel) relative to the average value in the middle of the convective zone. Black and dark greys indicate overabundances while white and light greys indicate underabundances. The figure shows a $2 \mathrm{D}$ vertical slice through the $3 \mathrm{D}$ computation domain. The thin dashed line indicates the position of the convective zone in the corresponding $1 \mathrm{~d}$ model, and the thick dot-dashed line shows the convective boundary in the $3 \mathrm{~d}$ simulation.

\section{Surface deformation}

The deviation from spherical symmetry is proportional to the rotation ratio $\omega=\Omega_{\text {surf }} / \Omega_{\text {crit }} 1$ In the frame of the Roche model, the equatorial radius is at most $R_{\text {eq,crit }}=\frac{3}{2} R_{\text {pol,crit }}$, with the reasonable assumption that $R_{\text {pol,crit }}=R_{\text {pol, } \omega=0}$. An oblateness has been observed by interferometry in the case of the rapidly rotating star Achernar ( $\alpha$ Eridani) for example, in agreement with the value of 1.5 (Domiciano de Souza et al., 2003; Vinicius et al., 2006, Carciofi et al., 2008).

Because of the deformation, the effective gravity in the Roche approximation becomes dependent on the rotation rate and the colatitude:

$$
\vec{g}_{\text {eff }}=\vec{g}_{\text {eff }}(\Omega, \theta)=\left(-\frac{G M}{r^{2}}+\Omega^{2} r \sin ^{2} \theta\right) \vec{e}_{r}+\Omega^{2} r \sin \theta \cos \theta \vec{e}_{\theta} .
$$

Subsequently, the flux inherits from this $\Omega-\theta$ dependence (e.g. Maeder, 1999):

$$
\vec{F}=\vec{F}(\Omega, \theta)=-\frac{L}{4 \pi G M^{\star}} \vec{g}_{\text {eff }}(\Omega, \theta),
$$

where $M^{\star}=M\left(1-\frac{\Omega^{2}}{2 \pi G \rho_{M}}\right)$. According to the Stefan-Boltzman law, we have $F=\sigma T^{4}$,

${ }^{1}$ We use here $\Omega_{\text {crit }}=\sqrt{\frac{8}{27} \frac{G M}{R_{\text {pol,crit }}^{3}}}$, where $R_{\text {pol,crit }}$ is the polar radius at the critical velocity. 


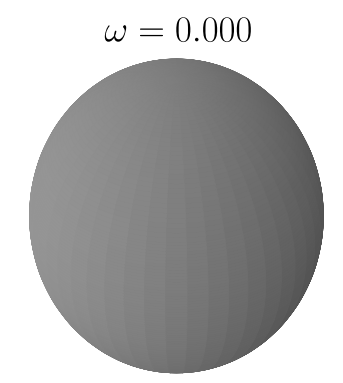

$\omega=0.750$

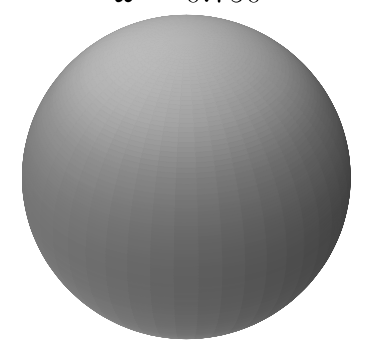

$\omega=0.500$

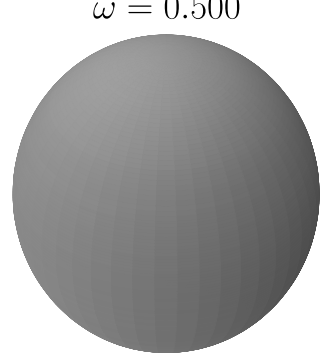

$\omega=0.990$

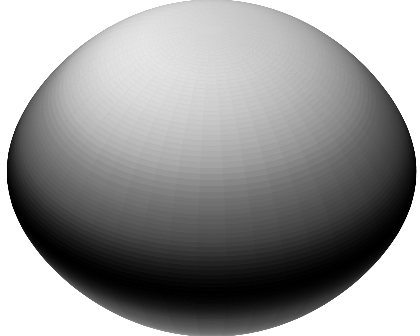

$T_{\text {eff }}(\theta) / T_{\text {eff,mean }}^{1.00}$

Figure 1.3 Effective temperature of rotating stars relative to the mean $T_{\text {eff }}$ for four different rotation rates $\omega=\Omega_{\text {surf }} / \Omega_{\text {crit }}$. The $T_{\text {eff }}(\theta)$ law is from Espinosa Lara and Rieutord (2011).

so the effective temperature also becomes dependent on $\Omega$ and $\theta$ (von Zeipel, 1924):

$$
T_{\mathrm{eff}}=\left[\frac{L}{4 \pi \sigma G M^{\star}} g_{\mathrm{eff}}(\Omega, \theta)\right]^{1 / 4} .
$$

Espinosa Lara and Rieutord (2011) have proposed a more general expression, valid also in the case of rapid rotation:

$$
T_{\mathrm{eff}}=\left(\frac{L}{4 \pi \sigma G M}\right)^{1 / 4} \sqrt{\frac{\tan \vartheta}{\tan \theta}} g_{\mathrm{eff}}^{1 / 4}
$$

where $\vartheta$ is the solution of the equation:

$$
\cos \vartheta+\ln \tan \frac{\vartheta}{2}=\frac{1}{3} \omega^{2} \tilde{r}^{3} \cos ^{3} \theta+\cos \theta+\ln \tan \frac{\theta}{2} .
$$

The latitude dependence of the temperature has been observed in interferometry for the star Altair ( $\alpha$ Aquilae, see Monnier et al., 2007) and the stars Alderamin and Rasalhague ( $\alpha$ Ceph and $\alpha$ Oph, see Zhao et al. 2009), with values compatible with the models.

Below $\omega=0.7$, the deformation remains insignificant (see Fig. 1.3), but above this value, the angle with which the stars are observed becomes an important parameter. A star observed pole-on will present a higher $L$ and $T_{\text {eff }}$ than the same star seen with an average 
inclination. In contrast, a star seen equator-on will appear dimmer and cooler (e.g. Georgy et al., 2014a). The stellar parameters deduced for such stars will be affected.

Hotter poles and cooler equators modify the mass flux ejected by the star, that gets a latitudinal dependence (Maeder and Meynet, 2000):

$$
\frac{\mathrm{d} \dot{M}(\theta)}{\mathrm{d} \sigma} \sim A(\alpha, k)\left(\frac{L}{4 \pi G M^{\star}}\right)^{\frac{1}{\alpha}-\frac{1}{8}} \frac{g_{\text {eff }}^{1-\frac{1}{8}}}{\left(1-\Gamma_{\Omega}(\theta)\right)^{\frac{1}{\alpha}-1}}
$$

where $\mathrm{d} \sigma$ is the surface unit, $A(\alpha, k)$ is an empirically determined function of the force multiplier parameters $\alpha$ and $k$ (Lamers et al. 1995), and $\Gamma_{\Omega}(\theta)$ is the local Eddington factor taking into account the effects of rotation:

$$
\Gamma_{\Omega}(\theta)=\frac{\kappa_{\mathrm{es}} L}{4 \pi c G M\left(1-\frac{\Omega^{2}}{2 \pi G \rho_{\mathrm{m}}}\right)}
$$

with $\kappa_{\mathrm{es}}$ the electron-scattering opacity, and $\rho_{\mathrm{m}}$ the internal average density. According to Georgy et al. (2011), for a nearly critically-rotating star, we have $\dot{M}(\mathrm{pol})=3.25 \dot{M}(\mathrm{eq})$. This anisotropy modifies the angular momentum budget, since the mass loss occurs preferentially in the region of the poles, which removes less angular momentum than what the equatorial regions would do (see also Müller and Vink, 2014).

\section{Transport processes}

In 1D, the transport of angular momentum is, according to Zahn (1992); Maeder and Zahn (1998):

$$
\rho \frac{\partial}{\partial t}\left(r^{2} \bar{\Omega}\right)_{M_{r}}=\underbrace{\frac{1}{5 r^{2}} \frac{\partial}{\partial r}\left(\rho r^{4} \bar{\Omega} U(r)\right)}_{\text {advective term }}+\underbrace{\frac{1}{r^{2}} \frac{\partial}{\partial r}\left(\rho D_{\mathrm{v}} r^{4} \frac{\partial \bar{\Omega}}{\partial r}\right)}_{\text {diffusive term }}
$$

where $U(r)$ is the function such that the radial component of the meridional circulation is $U(r) P_{2} \cos (\theta)$, and $D_{\mathrm{v}}$ the diffusion coefficient due to various mechanisms like convection or shear. The transport of chemical species has been shown to be satisfactorily approximated by a diffusion-only process (Chaboyer and Zahn, 1992) and can be expressed as:

$$
\rho \frac{\partial X_{i}}{\partial t}=\frac{1}{r^{2}} \frac{\partial}{\partial r}\left(\rho r^{2}\left(D_{\mathrm{v}}+D_{\text {eff }}\right) \frac{\partial X_{i}}{\partial r}\right)
$$

with $D_{\text {eff }}$ the diffusion coefficient due to meridional circulation and horizontal turbulence.

The implementation of rotation in the various codes existing on the market varies a lot from one to another. Some of them use a diffusion-only approximation to express the transport of angular momentum, as for the species. When the advective term is taken into account, three different expressions for the horizontal turbulence can be used (Zahn. 1992. Maeder 2003, Mathis et al., 2004), and two for the shear turbulence (Maeder, 1997, Talon and Zahn, 1997). Maeder et al. (2013) proposed also a global diffusion coefficient covering all the various instabilities triggered by rotation (GSF and Solberg-Høiland instabilities, 


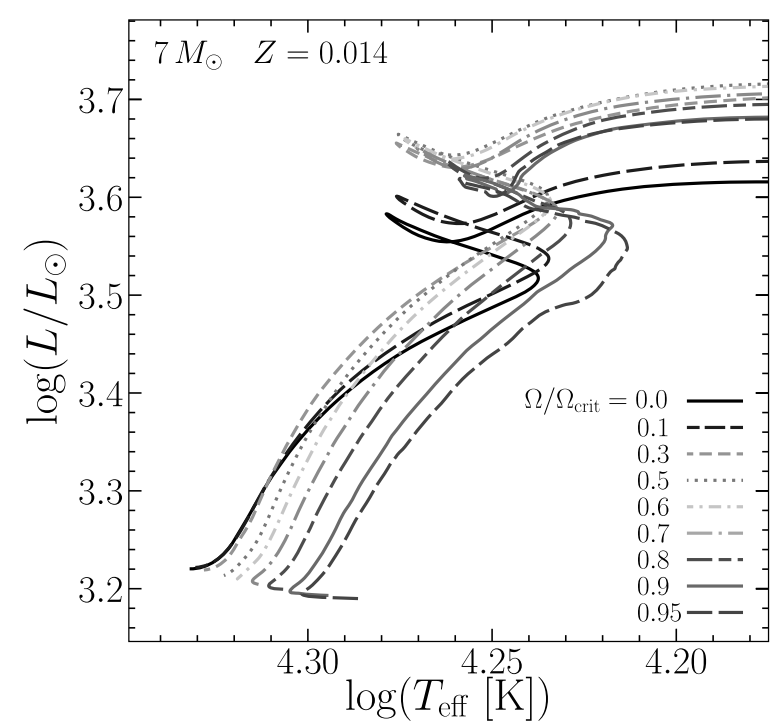

Figure 1.4 Main sequence tracks in the Hertzsprung-Russell diagram for models of $7 M_{\odot}$ with various initial rotation rates. (Models from Georgy et al. 2013).

thermohaline mixing, ...) and the interplay they have one with each others. The various ways of expressing the mixing process induced by rotation leads to large differences between the outputs of different codes, as highlighted by Chieffi and Limongi (2013). A study of the impact the different implementations have on the stellar evolution is presented in Meynet et al. (2013).

\section{Effects on the evolution}

Rotational mixing brings fresh fuel into the core, allowing it to grow further, hence resulting in longer lifetimes and a higher luminosity. However, the behaviour of a star is not monotonic with increasing rotation. At the beginning of the main sequence, the hydrostatic effects dominate and the star presents a slightly lower $L$ and $T_{\text {eff }}$. As the evolution proceeds, the mixing induces a slower shrinking of the core, increasing the luminosity, while the $T_{\text {eff }}$ decreases because of the modified chemical structure. Note that the effects of mixing are dominant for low and average rotation, while the rapid rotators show a composite behaviour between strong hydrostatic effects and mixing effects. The brightest star are therefore not the fastest ones, but the slightly-above-average rotators (see Fig. 1.4). In some extreme cases, the mixing during the main sequence is so strong that the star evolves quasi chemically homogeneously (usually at low metallicity, see Yoon et al., 2006, Szécsi] et al. 2015). In that case the star becomes extremely hot and compact, becoming a possible progenitor for long soft gamma-ray bursts (and gravitational waves, de Mink and Mandel, 2016. 


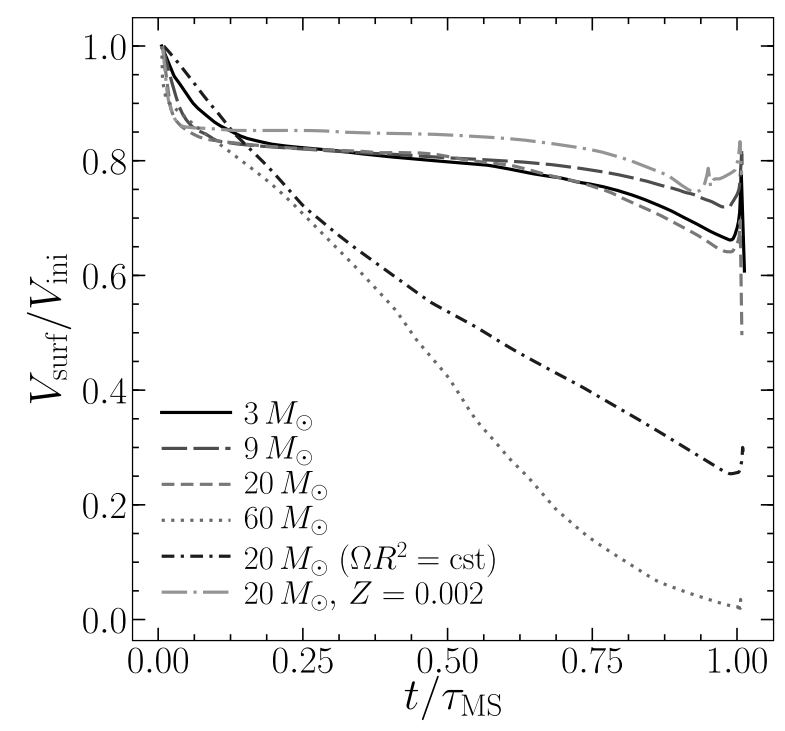

Figure 1.5 Evolution of the equatorial velocity (normalised to the initial one) during the main sequence for various models at $Z=0.014$ computed with an initial rotation ratio $\omega=0.50$.

\section{Evolution of the surface velocity}

If only the local conservation of angular momentum is considered, the surface velocity is expected to decrease during the main sequence, because of the natural expansion of the surface (see the short-dash-dotted curve in Fig. 1.5 for a $20 M_{\odot}$ model). On top of this natural evolution, two competing processes come into play: the mass loss (that removes angular momentum from the surface and triggers meridional currents), and the internal transport (that generally brings angular momentum from the centre to the surface). The strength of both the transport and the winds scales with the mass and metallicity, higher mass stars having stronger winds and more efficient transport, as do more metallic stars compared to low- $Z$ ones. The net result depends on their relative strength, as shown in Fig. 1.5. In the $3 M_{\odot}$ and the $9 M_{\odot}$ models (black solid and grey long-dashed curves respectively), the winds are negligible, the only difference is the strength of the transport, which is a little larger inside the $9 M_{\odot}$ model. The $20 M_{\odot}$ model (grey short-dashed curve) has a stronger transport, but the winds also become non-negligible, so the net result is a slight decrease of $V_{\text {surf }}$ during the main sequence. The case with no transport at all (local conservation of the angular momentum) is shown (dark grey, short-dashed-dotted curve) for comparison. In the $60 M_{\odot}$ model, the transport is very strong but the wind effect largely dominates and the star is very efficiently braked. Actually in that mass range, any comparison of rotation rates with observations should be considered as a check for the mass-loss rate used rather than a check for the treatment of rotation itself. A low metallicity weakens the internal transport but also the winds, as illustrated by the grey long-dashed-dotted curve in Fig. 1.5 
(20 $M_{\odot}$ model at $\left.Z=0.002\right)$, to be compared with the grey short-dashed one (same mass, $Z=0.014)$.

Note that the surface velocity and its ratio to the critical velocity $V / V_{\text {crit }}$ evolve differently: $V_{\text {crit }}=\sqrt{\frac{2}{3} \frac{G M}{R_{\text {pol }}}}$ decreases always during the main sequence, because the mass decreases and the radius inflates. Therefore, even if the surface velocity decreases with the evolution, the ratio to the critical velocity usually increases during the main sequence.

\subsection{Conclusion}

In this chapter, we have discussed some of the uncertainties inherent to the modelling of single massive stars. In the first section, we have shown how the inclusion of various massloss rates prescription can affect the evolution of massive stars. In particular, the mass loss during the red supergiant phase, which is still largely unknown, plays a key role for the subsequent evolution of the star and its final location in the Hertzsprung-Russell diagram.

Another key ingredient of stellar modelling is the treatment of convection. Here again, the difficulties of a correct treatment in classical 1d stellar evolution codes are a source of major shortcomings in our understanding of massive star life. The hopes for a better understanding and an improved modelling of convection come nowadays from multidimensional hydrodynamics simulations.

Finally, the implementation of rotation, which is suspected to play an important role as a mixing process inside massive stars, also suffers from uncertainties. The various possible ways of implementing rotation in stellar evolution codes make the prediction of simulations largely uncertain.

All of the uncertainties discussed above, related to single star evolution, also appear in the modelling of multiple systems, and they should be kept in mind when considering the results of any simulations involving stars.

\section{References}

Abbott, D. C. 1982. The theory of radiatively driven stellar winds. II - The line acceleration. ApJ, 259(Aug.), 282.

Adams, S. M., Kochanek, C. S., Gerke, J. R., Stanek, K. Z., and Dai, X. 2017. The search for failed supernovae with the Large Binocular Telescope: confirmation of a disappearing star. MNRAS, 468(July), 4968-4981.

Arnett, D., Meakin, C., and Young, P. A. 2009. Turbulent Convection in Stellar Interiors. II. The Velocity Field. ApJ, 690(Jan.), 1715-1729.

Arnett, W. D., Meakin, C., Viallet, M., Campbell, S. W., Lattanzio, J. C., and Mocák, M. 2015. Beyond Mixing-length Theory: A Step Toward 321D. ApJ, 809(Aug.), 30.

Battino, U., Pignatari, M., Ritter, C., Herwig, F., Denisenkov, P., Den Hartogh, J. W., Trappitsch, R., Hirschi, R., Freytag, B., Thielemann, F., and Paxton, B. 2016. Application of a Theory and Simulation-based Convective Boundary Mixing Model for AGB Star Evolution and Nucleosynthesis. ApJ, 827(Aug.), 30. 
Beasor, E. R., and Davies, B. 2016. The evolution of red supergiants to supernova in NGC 2100. MNRAS, 463(Dec.), 1269-1283.

Beasor, E. R., and Davies, B. 2017. The evolution of Red Supergiant mass-loss rates. ArXiv e-prints, Dec.

Bestenlehner, J. M., Gräfener, G., Vink, J. S., Najarro, F., de Koter, A., Sana, H., Evans, C. J., Crowther, P. A., Hénault-Brunet, V., Herrero, A., Langer, N., Schneider, F. R. N., Simón-Díaz, S., Taylor, W. D., and Walborn, N. R. 2014. The VLT-FLAMES Tarantula Survey. XVII. Physical and wind properties of massive stars at the top of the main sequence. $A \& A, \mathbf{5 7 0}$ (Oct.), A38.

Böhm-Vitense, E. 1958. Über die Wasserstoffkonvektionszone in Sternen verschiedener Effektivtemperaturen und Leuchtkräfte. Mit 5 Textabbildungen. ZAp, 46, 108.

Carciofi, A. C., Domiciano de Souza, A., Magalhães, A. M., Bjorkman, J. E., and Vakili, F. 2008. On the Determination of the Rotational Oblateness of Achernar. ApJ, 676(Mar.), L41.

Castor, J. I., Abbott, D. C., and Klein, R. I. 1975. Radiation-driven winds in Of stars. ApJ, 195(Jan.), 157-174.

Chaboyer, B., and Zahn, J.-P. 1992. Effect of horizontal turbulent diffusion on transport by meridional circulation. $A \& A, \mathbf{2 5 3}$ (Jan.), 173.

Chiavassa, A., Plez, B., Josselin, E., and Freytag, B. 2009. Radiative hydrodynamics simulations of red supergiant stars. I. interpretation of interferometric observations. $A \& A$, 506(Nov.), 1351-1365.

Chieffi, A., and Limongi, M. 2013. Pre-supernova Evolution of Rotating Solar Metallicity Stars in the Mass Range $13-120 M_{\odot}$ and their Explosive Yields. ApJ, 764(Feb.), 21.

Choplin, A., Ekström, S., Meynet, G., Maeder, A., Georgy, C., and Hirschi, R. 2017. Presupernova mixing in CEMP-no source stars. A\&A, 605(Sept.), A63.

Couch, S. M., Chatzopoulos, E., Arnett, W. D., and Timmes, F. X. 2015. The Threedimensional Evolution to Core Collapse of a Massive Star. ApJL, 808(July), L21.

Cristini, A., Meakin, C., Hirschi, R., Arnett, D., Georgy, C., Viallet, M., and Walkington, I. 2017. 3D hydrodynamic simulations of carbon burning in massive stars. MNRAS, 471(Oct.), 279-300.

Davis, A., Jones, S., and Herwig, F. 2017. Convective boundary mixing in a post-He core burning massive star model. ArXiv e-prints, Nov.

de Mink, S. E., and Mandel, I. 2016. The chemically homogeneous evolutionary channel for binary black hole mergers: rates and properties of gravitational-wave events detectable by advanced LIGO. MNRAS, 460(Aug.), 3545.

de Mink, S. E., Langer, N., Izzard, R. G., Sana, H., and de Koter, A. 2013. The Rotation Rates of Massive Stars: The Role of Binary Interaction through Tides, Mass Transfer, and Mergers. ApJ, 764(Feb.), 166.

Domiciano de Souza, A., Kervella, P., Jankov, S., Abe, L., Vakili, F., di Folco, E., and Paresce, F. 2003. The spinning-top Be star Achernar from VLTI-VINCI. A\&A, 407(Aug.), L47.

Eddington, A. S. 1925. Circulating currents in rotating stars. The Observatory, 48(Mar.), 73-75.

Eddington, A. S. 1926. The Internal Constitution of the Stars. Dover Publications Inc.

Eggenberger, P., Meynet, G., Maeder, A., Hirschi, R., Charbonnel, C., Talon, S., and Ekström, S. 2008. The Geneva stellar evolution code. Ap\&SS, 316(Aug.), 43-54.

Ekström, S., Georgy, C., Eggenberger, P., Meynet, G., Mowlavi, N., Wyttenbach, A., Granada, A., Decressin, T., Hirschi, R., Frischknecht, U., Charbonnel, C., and 
Maeder, A. 2012. Grids of stellar models with rotation. I. Models from 0.8 to $120 M_{\odot}$ at solar metallicity $(Z=0.014)$. A\&A, 537(Jan.), A146.

Eldridge, J. J., Izzard, R. G., and Tout, C. A. 2008. The effect of massive binaries on stellar populations and supernova progenitors. MNRAS, 384(Mar.), 1109-1118.

Endal, A. S., and Sofia, S. 1976. The evolution of rotating stars. I - Method and exploratory calculations for a 7-solar-mass star. ApJ, 210(Nov.), 184-198.

Espinosa Lara, F., and Rieutord, M. 2011. Gravity darkening in rotating stars. A\&A, 533(Sept.), A43.

Freytag, B., and Höfner, S. 2008. Three-dimensional simulations of the atmosphere of an AGB star. A\&A, 483(May), 571-583.

Freytag, B., Ludwig, H.-G., and Steffen, M. 1996. Hydrodynamical models of stellar convection. The role of overshoot in DA white dwarfs, A-type stars, and the Sun. $A \& A, \mathbf{3 1 3}$ (Sept.), 497-516.

Georgy, C. 2012. Yellow supergiants as supernova progenitors: an indication of strong mass loss for red supergiants? A\&A, 538(Feb.), L8.

Georgy, C. 2017 (Nov.). Evolution models of red supergiants. Pages 193-198 of: Eldridge, J. J., Bray, J. C., McClelland, L. A. S., and Xiao, L. (eds), The Lives and Death-Throes of Massive Stars. IAU Symposium, vol. 329.

Georgy, C., Meynet, G., and Maeder, A. 2011. Effects of anisotropic winds on massive star evolution. A\&A, 527(Mar.), A52.

Georgy, C., Ekström, S., Granada, A., Meynet, G., Mowlavi, N., Eggenberger, P., and Maeder, A. 2013. Populations of rotating stars. I. Models from 1.7 to $15 M_{\odot}$ at $Z=$ $0.014,0.006$, and 0.002 with $\Omega / \Omega_{\text {crit }}$ between 0 and $1 . A \& A, \mathbf{5 5 3}$ (May), A24.

Georgy, C., Granada, A., Ekström, S., Meynet, G., Anderson, R. I., Wyttenbach, A., Eggenberger, P., and Maeder, A. 2014a. Populations of rotating stars. III. SYCLIST, the new Geneva population synthesis code. A\&A, 566(June), A21.

Georgy, C., Saio, H., and Meynet, G. 2014b. The puzzle of the CNO abundances of $\alpha$ Cygni variables resolved by the Ledoux criterion. MNRAS, 439(Mar.), L6-L10.

Gräfener, G., Vink, J. S., de Koter, A., and Langer, N. 2011. The Eddington factor as the key to understand the winds of the most massive stars. Evidence for a $\Gamma$-dependence of Wolf-Rayet type mass loss. A\&A, 535(Nov.), A56.

Heger, A., Langer, N., and Woosley, S. E. 2000. Presupernova Evolution of Rotating Massive Stars. I. Numerical Method and Evolution of the Internal Stellar Structure. ApJ, 528(Jan.), 368-396.

Heger, A., Fryer, C. L., Woosley, S. E., Langer, N., and Hartmann, D. H. 2003. How Massive Single Stars End Their Life. ApJ, 591(July), 288.

Herwig, F. 2000. The evolution of AGB stars with convective overshoot. A\&A, 360(Aug.), 952-968.

Herwig, F., Freytag, B., Hueckstaedt, R. M., and Timmes, F. X. 2006. Hydrodynamic Simulations of He Shell Flash Convection. ApJ, 642(May), 1057-1074.

Jones, S., Hirschi, R., Nomoto, K., Fischer, T., Timmes, F. X., Herwig, F., Paxton, B., Toki, H., Suzuki, T., Martínez-Pinedo, G., Lam, Y. H., and Bertolli, M. G. 2013. Advanced Burning Stages and Fate of $8-10 M_{\odot}$ Stars. ApJ, 772(Aug.), 150.

Jones, S., Andrassy, R., Sandalski, S., Davis, A., Woodward, P., and Herwig, F. 2017. Idealized hydrodynamic simulations of turbulent oxygen-burning shell convection in $4 \pi$ geometry. MNRAS, 465(Mar.), 2991-3010.

Keszthelyi, Z., Puls, J., and Wade, G. A. 2017. Modeling the early evolution of massive OB stars with an experimental wind routine. The first bi-stability jump and the angular momentum loss problem. $A \& A, \mathbf{5 9 8}(\mathrm{Feb}$.), A4. 
Kippenhahn, R., and Weigert, A. 1990. Stellar Structure and Evolution. Stellar Structure and Evolution, XVI, 468 pp. 192 figs. Springer-Verlag Berlin Heidelberg New York. Also Astronomy and Astrophysics Library.

Kolmogorov, A. 1941. The Local Structure of Turbulence in Incompressible Viscous Fluid for Very Large Reynolds' Numbers. Akademiia Nauk SSSR Doklady, 30, 301-305.

Kolmogorov, A. N. 1962. A refinement of previous hypotheses concerning the local structure of turbulence in a viscous incompressible fluid at high Reynolds number. Journal of Fluid Mechanics, 13, 82-85.

Kudritzki, R. P. 2002. Line-driven Winds, Ionizing Fluxes, and Ultraviolet Spectra of Hot Stars at Extremely Low Metallicity. I. Very Massive O Stars. ApJ, 577(Sept.), 389.

Kudritzki, R.-P., and Puls, J. 2000. Winds from Hot Stars. ARA\&A, 38, 613-666.

Lamers, H. J. G. L. M., Snow, T. P., and Lindholm, D. M. 1995. Terminal Velocities and the Bistability of Stellar Winds. ApJ, 455(Dec.), 269.

Lucy, L. B., and Solomon, P. M. 1970. Mass Loss by Hot Stars. ApJ, 159(Mar.), 879-+.

Maeder, A. 1975. Stellar evolution. IV - Evolution of a star of 1.5 solar masses from the main-sequence to the red-giant branch with and without overshooting from convective core. $A \& A, 43$ (Sept.), 61-69.

Maeder, A. 1997. Stellar evolution with rotation. II. A new approach for shear mixing. $A \& A$, 321(May), 134.

Maeder, A. 1999. Stellar evolution with rotation IV: von Zeipel's theorem and anisotropic losses of mass and angular momentum. A\&A, 347(July), 185-193.

Maeder, A. 2003. Stellar rotation: Evidence for a large horizontal turbulence and its effects on evolution. A\&A, 399(Feb.), 263.

Maeder, A. 2009. Physics, Formation and Evolution of Rotating Stars. Berlin Heidelberg: Springer Verlag.

Maeder, A., and Meynet, G. 2000. Stellar evolution with rotation. VI. The Eddington and Omega -limits, the rotational mass loss for OB and LBV stars. A\&A, 361(Sept.), 159.

Maeder, A., and Zahn, J.-P. 1998. Stellar evolution with rotation. III. Meridional circulation with MU -gradients and non-stationarity. A\&A, 334(June), 1000.

Maeder, A., Meynet, G., Lagarde, N., and Charbonnel, C. 2013. The thermohaline, Richardson, Rayleigh-Taylor, Solberg-Høiland, and GSF criteria in rotating stars. $A \& A$, 553(May), A1.

Magic, Z., Collet, R., Asplund, M., Trampedach, R., Hayek, W., Chiavassa, A., Stein, R. F., and Nordlund, А̊. 2013. The Stagger-grid: A grid of 3D stellar atmosphere models. I. Methods and general properties. $A \& A, 557$ (Sept.), A26.

Martins, F., and Palacios, A. 2013. A comparison of evolutionary tracks for single Galactic massive stars. $A \& A, \mathbf{5 6 0}$ (Dec.), A16.

Mathis, S., Palacios, A., and Zahn, J.-P. 2004. On shear-induced turbulence in rotating stars. $A \& A, \mathbf{4 2 5}$ (Oct.), 243.

Mauron, N., and Josselin, E. 2011. The mass-loss rates of red supergiants and the de Jager prescription. $A \& A, \mathbf{5 2 6}$ (Feb.), A156.

Meakin, C. A., and Arnett, D. 2007. Turbulent Convection in Stellar Interiors. I. Hydrodynamic Simulation. ApJ, 667(Sept.), 448-475.

Meynet, G., Ekström, S., Maeder, A., Eggenberger, P., Saio, H., Chomienne, V., and Haemmerlé, L. 2013. Models of Rotating Massive Stars: Impacts of Various Prescriptions. Page 3 of: Goupil, M., Belkacem, K., Neiner, C., Lignières, F., and Green, J. J. (eds), Studying Stellar Rotation and Convection. Lecture Notes in Physics, vol. 865. 
Meynet, G., Chomienne, V., Ekström, S., Georgy, C., Granada, A., Groh, J., Maeder, A., Eggenberger, P., Levesque, E., and Massey, P. 2015. Impact of mass-loss on the evolution and pre-supernova properties of red supergiants. A\&A, 575(Mar.), A60.

Mocák, M., Meakin, C., Viallet, M., and Arnett, D. 2014. Compressible Hydrodynamic Mean-Field Equations in Spherical Geometry and their Application to Turbulent Stellar Convection Data. ArXiv e-prints, Jan.

Mokiem, M. R., de Koter, A., Vink, J. S., Puls, J., Evans, C. J., Smartt, S. J., Crowther, P. A., Herrero, A., Langer, N., Lennon, D. J., Najarro, F., and Villamariz, M. R. 2007. The empirical metallicity dependence of the mass-loss rate of $\mathrm{O}$ - and early B-type stars. $A \& A, \mathbf{4 7 3}$ (Oct.), 603.

Monnier, J. D., Zhao, M., Pedretti, E., Thureau, N., Ireland, M., Muirhead, P., Berger, J.P., Millan-Gabet, R., Van Belle, G., ten Brummelaar, T., McAlister, H., Ridgway, S., Turner, N., Sturmann, L., Sturmann, J., and Berger, D. 2007. Imaging the Surface of Altair. Science, 317(July), 342.

Moravveji, E., Aerts, C., Pápics, P. I., Triana, S. A., and Vandoren, B. 2015. Tight asteroseismic constraints on core overshooting and diffusive mixing in the slowly rotating pulsating B8.3V star KIC 10526294. A\&A, 580(Aug.), A27.

Müller, B., Viallet, M., Heger, A., and Janka, H.-T. 2016. The Last Minutes of Oxygen Shell Burning in a Massive Star. ApJ, 833(Dec.), 124.

Müller, P. E., and Vink, J. S. 2014. Rotating massive O stars with non-spherical 2D winds. $A \& A, \mathbf{5 6 4}$ (Apr.), A57.

Najarro, F., Hanson, M. M., and Puls, J. 2011. L-band spectroscopy of Galactic OB-stars. $A \& A, \mathbf{5 3 5}$ (Nov.), A32.

O'Connor, E., and Ott, C. D. 2011. Black Hole Formation in Failing Core-Collapse Supernovae. ApJ, 730(Apr.), 70.

Paxton, B., Bildsten, L., Dotter, A., Herwig, F., Lesaffre, P., and Timmes, F. 2011. Modules for Experiments in Stellar Astrophysics (MESA). ApJS, 192(Jan.), 3.

Paxton, B., Cantiello, M., Arras, P., Bildsten, L., Brown, E. F., Dotter, A., Mankovich, C., Montgomery, M. H., Stello, D., Timmes, F. X., and Townsend, R. 2013. Modules for Experiments in Stellar Astrophysics (MESA): Planets, Oscillations, Rotation, and Massive Stars. ApJS, 208(Sept.), 4.

Paxton, B., Marchant, P., Schwab, J., Bauer, E. B., Bildsten, L., Cantiello, M., Dessart, L., Farmer, R., Hu, H., Langer, N., Townsend, R. H. D., Townsley, D. M., and Timmes, F. X. 2015. Modules for Experiments in Stellar Astrophysics (MESA): Binaries, Pulsations, and Explosions. ApJS, 220(Sept.), 15.

Paxton, B., Schwab, J., Bauer, E. B., Bildsten, L., Blinnikov, S., Duffell, P., Farmer, R., Goldberg, J. A., Marchant, P., Sorokina, E., Thoul, A., Townsend, R. H. D., and Timmes, F. X. 2017. Modules for Experiments in Stellar Astrophysics (MESA): Convective Boundaries, Element Diffusion, and Massive Star Explosions. ArXiv e-prints, Oct.

Petrovic, J., Langer, N., Yoon, S.-C., and Heger, A. 2005. Which massive stars are gammaray burst progenitors? A\&A, 435(May), 247-259.

Podsiadlowski, P., Joss, P. C., and Hsu, J. J. L. 1992. Presupernova evolution in massive interacting binaries. ApJ, 391(May), 246-264.

Puls, J., Vink, J. S., and Najarro, F. 2008. Mass loss from hot massive stars. A\&A Rev., 16(Dec.), 209-325.

Rauw, G., Hervé, A., Nazé, Y., González-Pérez, J. N., Hempelmann, A., Mittag, M., Schmitt, J. H. M. M., Schröder, K.-P., Gosset, E., Eenens, P., and Uuh-Sonda, J. M. 
2015. Simultaneous X-ray and optical spectroscopy of the Oef supergiant $\lambda$ Cephei. $A \& A, \mathbf{5 8 0}$ (Aug.), A59.

Saio, H., Georgy, C., and Meynet, G. 2013. Evolution of blue supergiants and $\alpha$ Cygni variables: puzzling CNO surface abundances. MNRAS, 433(Aug.), 1246-1257.

Sana, H., de Mink, S. E., de Koter, A., Langer, N., Evans, C. J., Gieles, M., Gosset, E., Izzard, R. G., Le Bouquin, J.-B., and Schneider, F. R. N. 2012. Binary Interaction Dominates the Evolution of Massive Stars. Science, 337(July), 444-.

Sana, H., de Koter, A., de Mink, S. E., Dunstall, P. R., Evans, C. J., Hénault-Brunet, V., Maíz Apellániz, J., Ramírez-Agudelo, O. H., Taylor, W. D., Walborn, N. R., Clark, J. S., Crowther, P. A., Herrero, A., Gieles, M., Langer, N., Lennon, D. J., and Vink, J. S. 2013. The VLT-FLAMES Tarantula Survey. VIII. Multiplicity properties of the O-type star population. $A \& A, \mathbf{5 5 0}$ (Feb.), A107.

Smartt, S. J., Eldridge, J. J., Crockett, R. M., and Maund, J. R. 2009. The death of massive stars - I. Observational constraints on the progenitors of Type II-P supernovae. MNRAS, 395(May), 1409-1437.

Sukhbold, T., and Woosley, S. E. 2014. The Compactness of Presupernova Stellar Cores. ApJ, 783(Mar.), 10.

Szécsi, D., Langer, N., Yoon, S.-C., Sanyal, D., de Mink, S., Evans, C. J., and Dermine, T. 2015. Low-metallicity massive single stars with rotation. Evolutionary models applicable to I Zwicky 18. A\&A, 581(Sept.), A15.

Talon, S., and Zahn, J.-P. 1997. Anisotropic diffusion and shear instabilities. $A \& A$, 317(Feb.), 749.

Šurlan, B., Hamann, W.-R., Aret, A., Kubát, J., Oskinova, L. M., and Torres, A. F. 2013. Macroclumping as solution of the discrepancy between $\mathrm{H} \alpha$ and $\mathrm{P} v$ mass loss diagnostics for O-type stars. $A \& A, \mathbf{5 5 9}$ (Nov.), A130.

van den Heuvel, E. P. J. 1975. Modes of mass transfer and classes of binary X-ray sources. ApJL, 198(June), L109.

van Loon, J. T., Cioni, M.-R. L., Zijlstra, A. A., and Loup, C. 2005. An empirical formula for the mass-loss rates of dust-enshrouded red supergiants and oxygen-rich Asymptotic Giant Branch stars. A\&A, 438(July), 273-289.

Vanbeveren, D. 1991. Massive close binaries - Observations and evolutionary computations. Space Sci. Rev., 56(May), 249-311.

Vanbeveren, D., De Donder, E., van Bever, J., van Rensbergen, W., and De Loore, C. 1998. The WR and O-type star population predicted by massive star evolutionary synthesis. New A, 3(Nov.), 443-492.

Viallet, M., Meakin, C., Arnett, D., and Mocák, M. 2013. Turbulent Convection in Stellar Interiors. III. Mean-field Analysis and Stratification Effects. ApJ, 769(May), 1.

Vinicius, M. M. F., Zorec, J., Leister, N. V., and Levenhagen, R. S. 2006. alpha Eridani: rotational distortion, stellar and circumstellar activity. $A \& A, \mathbf{4 4 6}$ (Feb.), 643.

Vink, J. S. 2006 (Dec.). Massive star feedback - from the first stars to the present. Page 113 of: Lamers, H. J. G. L. M., Langer, N., Nugis, T., and Annuk, K. (eds), Stellar Evolution at Low Metallicity: Mass Loss, Explosions, Cosmology. Astronomical Society of the Pacific Conference Series, vol. 353.

Vink, J. S., de Koter, A., and Lamers, H. J. G. L. M. 2000. New theoretical mass-loss rates of $\mathrm{O}$ and $\mathrm{B}$ stars. A\&A, 362(Oct.), 295-309.

Vink, J. S., de Koter, A., and Lamers, H. J. G. L. M. 2001. Mass-loss predictions for O and B stars as a function of metallicity. A\&A, 369(Apr.), 574-588.

Vink, J. S., Muijres, L. E., Anthonisse, B., de Koter, A., Gräfener, G., and Langer, N. 2011. Wind modelling of very massive stars up to 300 solar masses. A\&A, $\mathbf{5 3 1}$ (July), A132. 
von Zeipel, H. 1924. The radiative equilibrium of a rotating system of gaseous masses. MNRAS, 84(June), 665.

Walmswell, J. J., and Eldridge, J. J. 2012. Circumstellar dust as a solution to the red supergiant supernova progenitor problem. MNRAS, 419(Jan.), 2054-2062.

Woodward, P. R., Herwig, F., and Lin, P.-H. 2015. Hydrodynamic Simulations of H Entrainment at the Top of He-shell Flash Convection. ApJ, 798(Jan.), 49.

Yoon, S.-C., Langer, N., and Norman, C. 2006. Single star progenitors of long gamma-ray bursts. I. Model grids and redshift dependent GRB rate. $A \& A, \mathbf{4 6 0}$ (Dec.), 199.

Yusof, N., Hirschi, R., Meynet, G., Crowther, P. A., Ekström, S., Frischknecht, U., Georgy, C., Abu Kassim, H., and Schnurr, O. 2013. Evolution and fate of very massive stars. MNRAS, 433(Aug.), 1114-1132.

Zahn, J.-P. 1991. Convective penetration in stellar interiors. A\&A, 252(Dec.), 179-188.

Zahn, J.-P. 1992. Circulation and turbulence in rotating stars. $A \& A, \mathbf{2 6 5}$ (Nov.), 115.

Zhao, M., Monnier, J. D., Pedretti, E., Thureau, N., Mérand, A., ten Brummelaar, T., McAlister, H., Ridgway, S. T., Turner, N., Sturmann, J., Sturmann, L., Goldfinger, P. J., and Farrington, C. 2009. Imaging and Modeling Rapidly Rotating Stars: $\alpha$ Cephei and $\alpha$ Ophiuchi. ApJ, 701(Aug.), 209. 